\title{
Trichothiodystrophy with sideroblastic anaemia and developmental delay
}

Sally A Lynch, David de Berker, Alan R Lehmann, Rodney J Pollitt, Michael M Reid, William H Lamb

\begin{abstract}
A patient with sideroblastic anaemia, development delay, and trichothiodystrophy is presented. Trichothiodystrophy is a feature of several autosomal recessive diseases. Photosensitivity, failure to thrive, and developmental delay are commonly observed in affected cases. $X$ linked inheritance accounts for the bulk of cases with sideroblastic anaemia. This case highlights the importance of routine hair microscopy in cases of atypical ectodermal dysplasia.

(Arch Dis Child 1995; 73: 249-251)
\end{abstract}

Keywords: trichothiodystrophy, sideroblastic anaemia, transcription factor, DNA repair defect.

Trichothiodystrophy (TTD) is a feature of several neurocutaneous conditions. The spectrum of symptoms and signs associated with TTD is extensive. ${ }^{1}$ An association between sideroblastic anaemia and TTD has not been reported previously.

\section{Case report}

The proband was born at term weighing $2310 \mathrm{~g}$ (<3rd centile) to unrelated parents.

Department of Human Genetics, 19/20

Claremont Place,

Newcastle upon Tyne NE2 4AA

S A Lynch

Royal Victoria

Infirmary, Newcastle upon Tyne,

Department of

Dermatology

$\mathrm{D}$ de Berker

\section{Department of \\ Haematology}

M M Reid

MRC Cell Mutation Unit, University of Sussex, Brighton A R Lehmann

Neonatal Screening Laboratory, The Children's Hospital, Sheffield

R J Pollit

Department of Paediatrics, Bishop Auckland General Hospital

W H Lamb

Correspondence and reprint requests to: Dr Lynch.

Accepted 17 May 1995
His mother was on bromocriptine for a prolactinoma throughout the pregnancy. At 3 months of age he was admitted to hospital with failure to thrive and a heart murmur. The heart murmur was secondary to sideroblastic anaemia. At 3 years of age, his weight, height, and head circumference were all $<3$ rd centile. Hair was absent from the frontal and temporal regions and sparse on the scalp and eyebrows (see fig 1). He had severe dental caries and a single fused lower central incisor. Nails and skin were normal. There was no history of cutaneous photosensitivity. At age 3 his gross motor and social development were six months behind his chronological age.

Investigations, at age 3 months, had shown a haemoglobin concentration of $63 \mathrm{~g} / \mathrm{l}$, with a mean corpuscular volume of $66.8 \mathrm{fl}$, and a normal white blood cell count. His blood film showed marked anisocytosis, poikilocytes, stippled red cells, and occasional small nucleated red cells. Bone marrow aspirate revealed a cellular marrow with erythroid hyperplasia. Dyserythropoiesis was observed with occasional binucleate red cells but no internuclear chromatin bridging: a feature one would expect in congenital dyserythropoietic anaemias. Iron staining showed that approximately $20 \%$ of the cells were ring sideroblasts. A diagnosis of sideroblastic anaemia was made. His haemoglobin returned to normal after a blood transfusion and treatment with pyridoxine.

Light microscopy showed pale floppy hairs with trichorrhexis nodes and brush ends. Scanning electron microscopy demonstrated loss of organised cuticular structure and ribbon like morphology of the hair shaft (see fig 2). Polarised light microscopy elicited the tiger tail appearance of alternating light and dark transverse bands associated with trichothiodystrophy. Hair amino acid analysis revealed low cystine, proline, threonine, and serine concentrations and increased concentrations of aspartic acid, lysine, leucine, and alanine. These findings are consistent with a diagnosis of TTD.

Dental examination revealed only three lower incisors with a fused left lower $A B$ incisor. Echocardiography and a skeletal survey were normal.

Chromosomal analysis revealed a normal male 46,XY karyotype; in particular no abnormality of Xp11.21 was identified.

Studies of cultured fibroblasts revealed no evidence of a DNA repair defect.

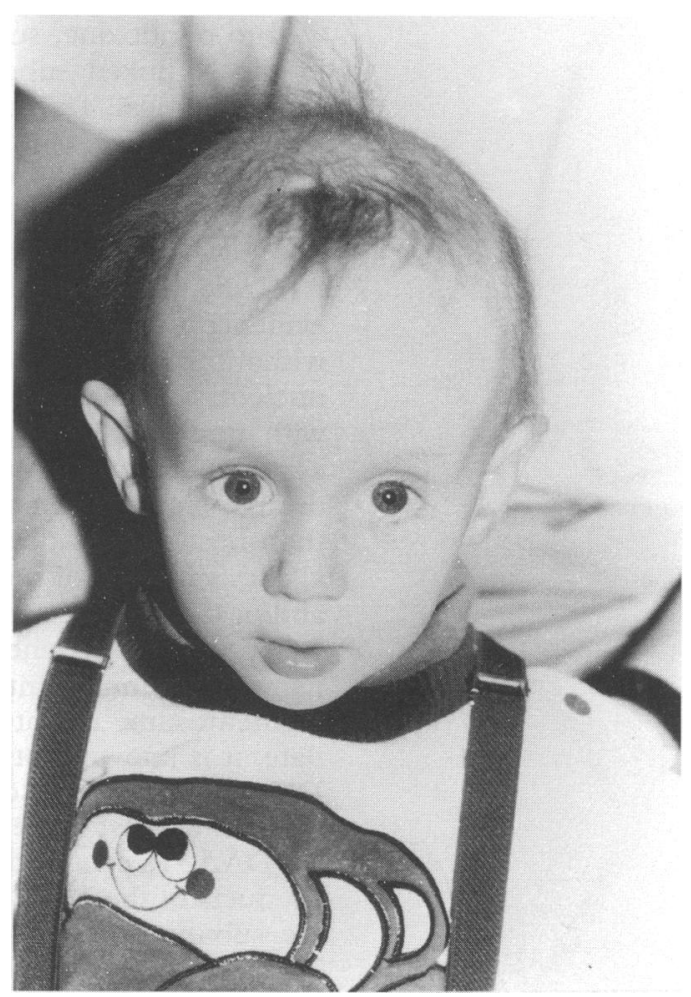

Figure 1 Photography of case showing sparse hair on the scalp and eyebrows. 


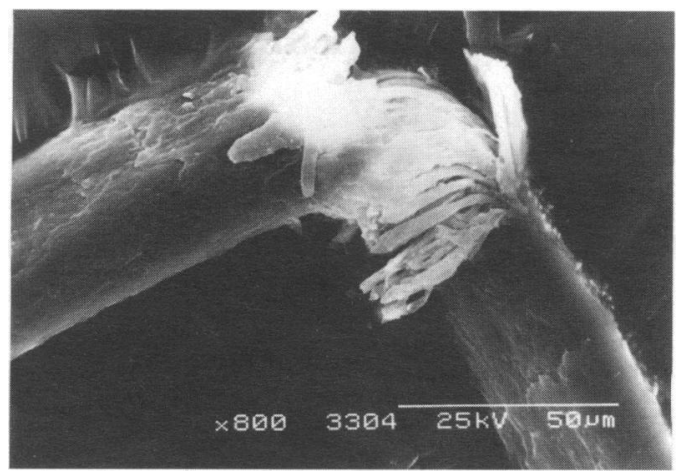

Figure 2 Scanning electron microscopy of a trichorrhexis node. The flattened ribbon-like morphology of the hair is demonstrated.

\section{Discussion}

The sideroblastic anaemias are a group of genetic or acquired disorders characterised by the presence of numerous ring sideroblasts and severe dyserythropoieses. There is one reported case of acquired sideroblastic anaemia associated with bromocriptine treatment (Committee on Safety of Medicines, personal communication). There is no evidence to support an association between bromocriptine treatment in pregnancy and sideroblastic anaemia in a child. There was also no family history of sideroblastic anaemia in the family and neither parent had any abnormalities on their blood film. X linked inheritance accounts for the bulk of the hereditary congenital forms of sideroblastic anaemia, although autosomal dominant, recessive, and mitochondrial inheritance have been reported. ${ }^{2}$ Mutations in the erythroid aminolevulinic acid synthase (ALAS2) gene at $\mathrm{Xp11.21}$ have been identified in patients with pyridoxine responsive $\mathrm{X}$ linked sideroblastic anaemia. ${ }^{3}$ The boy reported here responded well to pyridoxine, suggesting an abnormality in the $\mathrm{X}$ linked subgroup, although he had some atypical features. Normally, affected males do not present until childhood or later.

Patients with TTD can be broadly divided into two main groups depending on whether or not they have evidence of a DNA repair defect. As a rule, those with skin photosensitivity have evidence of a DNA repair defect and those without photosensitivity do not. Defects in nucleotide excision repair can be associated with three different genetic disorders: TTD, xeroderma pigmentosum, and Cockayne's syndrome. Complementation analysis has shown that most cases within this group of TTD are allelic variants of xeroderma pigmentosum and/or Cockayne's syndrome and that they are genetically heterogeneous. Interestingly malignancy, an important feature of xeroderma pigmentosum, is not a feature of TTD. To date, it is known that TTD, associated with a DNA repair defect, can be caused by mutations in at least three genes, XPB, XPD, and TTDA. ${ }^{4} 5$ Recent findings have shown that the products of the XPB, XPD, and TTDA genes are subunits of the basal transcription factor TFIIH. ${ }^{4}$ Thus, TFIIH has a dual role in the cell, as a DNA repair protein and as a basal transcription factor. Basal transcription factors are required for expression of all genes. It has been suggested that most of the clinical features of TTD result from a subtle defect in transcription caused by mutations altering TFIIH, rather than from defects in DNA repair. It is postulated that subtle deficiencies in the transcriptional apparatus have effects that are confined to specific genes, such as those involved in hair shaft development. ${ }^{4}$ Unfortunately, the biochemical pathway of hair amino acid metabolism is poorly understood and therefore the identities of the genes involved remain unknown. There is no evidence from linkage data nor from mutational analysis confirming or excluding linkage to XPB, XPD, or TTDA in families with TTD and normal nucleotide excision repair. It is possible that certain mutations in XPB, XPD, and TTDA cause TTD without disrupting DNA repair. The precise effects of a subtle transcriptional defect might vary significantly, depending on the nature of the mutation and on the genetic background of the affected individual. This could provide an explanation for the heterogeneity of symptoms associated with TTD, and could account for the sideroblastic anaemia seen in our patient. This idea of a genetic disorder secondary to transcription errors is not new: the $\alpha$-thalassemia mental retardation syndrome is an $\mathrm{X}$ linked condition with features of $\alpha$-thalassemia secondary to down regulation of the $\alpha$-globin gene on chromosome $16 .^{6}$

It is conceivable that the combination of TTD and sideroblastic anaemia in this boy is a complete coincidence. However, if they are related, autosomal recessive inheritance is likely, although there remains the possibility that our case represents an $\mathrm{X}$ linked variant of TTD. A review of the literature revealed one other case of an $\mathrm{X}$ linked condition in association with TTD. The case report describes a boy with TTD and atypical ornithine carbamyltransferase deficiency. ${ }^{7}$ The gene for ornithine carbamyltransferase maps to $\mathrm{Xp} 21.1$ far from the ALAS2 locus at Xp11.21. A deletion of this entire region would be easily picked up on cytogenetic analysis making the possibility of a contiguous gene syndrome unlikely.

Further elucidation of the biochemical pathways are needed in order to determine the presence or otherwise of an X linked enzyme essential for normal hair morphology. It may be that the clinical phenotype and laboratory profile of genetic disorders secondary to errors in transcription are distinct from the classic presentation. Offering prenatal diagnosis for genetic disorders with atypical presentations may be hazardous as the condition may be secondary to mutations in regulatory genes and not due to a mutation in the target gene. Further studies are ongoing in order to characterise the genetic basis of the condition in this case.

1 Itin PH, Pittelkow MR. Trichothiodystrophy: review of sulfur-deficient brittle hair syndromes and association with the ectodermal dysplasias. F Am Acad Dermatol 1990; 22 705-17.

2 McKusick VA. 301300 Congenital sideroblastic anaemia Mendelian inheritance in man. 11th Ed. Baltimore: Johns Hopkins University Press, 1994: 2308-10. 
3 Cotter PD, Baumann M, Bishop DF. Enzymatic defect in $\mathrm{X}$-linked sideroblastic anaemia: molecular evidence for erythroid delta-aminolevulinate synthase deficiency. Proc Natl Acad Sci USA 1992; 89: 4028-36.

4 Bootsma D, Hoeijmakers JHJ. Engagement with transcription. Nature 1993; 363: 114-5.

5 Stefanini M, Vermeulen W, Weeda G, et al. A new nucleotide-excision-repair gene associated with the disorder trichothiodystrophy. Am $\mathcal{F}$ Hum Genet 1993; 53: 817-21. 6 Gibbons RJ, Suthers GK, Wilkie AOM, et al. X-linked $\alpha$-thalassemia/mental retardation (ATR-X) syndrome: localisation to $\mathrm{Xq} 12-\mathrm{q} 21.31$ by $\mathrm{X}$ inactivation and linkage analysis. Am f Hum Genet 1992; 51: 1136-49.

7 Hordinsky MK, Briden B, Berry SA. Friable hair, urea cycle dysfunction and trichothiodystrophy. Curr Probl Dermatol 1987; 17: 52-60.

\title{
Growth hormone deficiency and empty sella in DIDMOAD syndrome: an endocrine study
}

\author{
Ashraf T Soliman, Bhasker Bappal, Assim Darwish, Anna Rajab, Maurice Asfour
}

\begin{abstract}
Two girls with DIDMOAD syndrome are presented. One also had severe megaloblastic-sideroblastic anaemia and the other several neurological manifestations. Both were short with defective growth hormone secretion. Computed tomography revealed empty sella in both girls; one had widespread atrophic cortical and cerebellar changes. High doses of thiamine improved the anaemia in the first case, increased $C$ peptide secretion in both, but had no effect on the neurological abnormalities.

(Arch Dis Child 1995; 73: 251-253)
\end{abstract}

Keywords: DIDMOAD syndrome, growth hormone, empty sella, insulin.

DIDMOAD syndrome, also known as Wolfram's syndrome, is an autosomal recessive condition with diabetes insipidus, diabetes mellitus, optic atrophy, and sensorineural deafness. These four cardinal features of DIDMOAD syndrome may be accompanied by a wide variety of other abnormalities affecting the nervous system and endocrine glands. These manifestations may be explained by a gradual neuronal degenerative process. $^{1}$ A sideroblastic and megaloblastic anaemia

Royal Hospital, Muscat, Oman, Departments of Paediatrics and Endocrinology A T Soliman B Bappal A Rajab

Department of Radiology A Darwish

National Diabetes Centre

M Asfour

Correspondence to: Dr Ashraf T Soliman Paediatric Endocrinology, Royal Hospital, National Diabetes Centre, PO Box 1331 Seeb, Muscat, Code III, Oman.

Accepted 11 May 1995 has been reported and treatment with high dose thiamine has improved the anaemia in these subjects. ${ }^{2}$ An inherited abnormality of thiamine metabolism has been suggested as responsible for this multisystem degenerative disorder. $^{3}$

This study describes the early involvement of the pituitary gland in the degenerative process contributing to growth delay in two Omani girls with DIDMOAD syndrome.

\section{Case reports}

CASE 1

Patient 1 was born at term to consanguineous parents after a full term normal delivery. She initiated presented with severe pallor and failure to thrive at the age of 22 months. Her elder sister, who was deaf and diabetic, died at the age of 7 years after an attack of profound and sustained hypoglycaemia due to administration of an excessive dose of insulin by her father at home. On admission she was severely anaemic with multiple bruises, bleeding gums, haematuria, petechiae, hepatomegaly, and mild splenomegaly. She had bilateral sensorineural deafness confirmed by auditory evoked responses and bilateral partial optic atrophy. The table presents the haematological findings showing severe anaemia with moderate macrocytosis, thrombocytopenia, and leucopenia. Haemoglobin electrophoresis, and measurement of her glucose-6-phosphate dehydrogenase, and serum electrolytes, urea, and creatinine concentrations, and liver function gave normal results. The direct and indirect Coombs test and Ham's test with sucrose were negative. Serum iron, iron binding capacity, ferritin, serum B12, and folate were normal. Bone marrow cellularity was normal with megaloblastic erythropoiesis. TORCH screen and hepatitis surface antigen were negative. Insulin was started after an abnormal oral glucose tolerance test. Glycated haemoglobin $\left(\mathrm{HbA} 1_{\mathrm{C}}\right)$ was $8 \cdot 7 \%$, fasting serum $\mathrm{C}$ peptide concentrations were $<0.8 \mu \mathrm{g} / 1$ before and six minutes after intravenous glucagon $(0.1 \mathrm{mg} / \mathrm{kg})$, and insulin antibodies were not detected in the serum. She was maintained on two daily doses of insulin and oral thiamine, $50 \mathrm{mg} /$ day. All the haematological abnormalities resolved within four weeks. Her basal and glucagon stimulated serum C peptide concentrations increased significantly after six months. At the age of 4.5 years her parents discontinued thiamine for three months against medical advice. Subsequently she was admitted with severe anaemia, purpura, and hyperglycaemia. Her $\mathrm{HbAl}_{C}$ was $9 \cdot 1 \%$. She again responded to thiamine and insulin. A water deprivation test revealed urine and serum osmolality of 320 and $290 \mathrm{mmol} / \mathrm{kg}$ respectively at 0 hours and 420 and 307 $\mathrm{mmol} / \mathrm{kg}$ after eight hours. Three hours after desmopressin (DDAVP, Ferring) $20 \mu \mathrm{g}$ intranasally, urine osmolality increased to $598 \mathrm{mmol} / \mathrm{kg}$ and plasma osmolality decreased to $289 \mathrm{mmol} / \mathrm{kg}$, consistent with 